HAL JFS 55 2015b/

Sablayrolles Jean-François (USPC et laboratoire HTL UMR 7597)

« Néologismes ludiques : études morphologique et énonciativo-pragmatique »

dans Esme Winter-Froemel et Angelika Zirker (éds), Enjeux du jeu de mots. Perspectives linguistiques et littéraires

Berlin, Boston, De Gruyter, p. 189-216

DOI: https://doi.org/10.1515/9783110408348

\title{
Résumé
}

Bien d'autres raisons que la nomination de nouveaux objets et concepts techniques et scientifiques expliquent l'émergence des néologismes. La fonction ludique n'est pas la moindre. Elle recourt à des matrices lexicogéniques privilégiées et elle se diversifie en divers types d'effets dans l'interlocution. Du point de vue morphologique, ce sont les procédés extragrammaticaux qui sont particulièrement activés (mot-valisation en particulier mais aussi paronymie, fausses coupes, innovations flexionnelles, etc.). Quand il s'agit de matrices régulières (suffixation et composition), on remarque des écarts entre les éléments qui sont mis en œuvre (hybrides, mots ou formants relevant de registres différents, etc.). D'un point de vue énonciatif et pragmatique, ces néologismes apparaissent dans des situations diverses (de l'hapax conversationnel aux néologismes qui se diffusent en passant par des situations intermédiaires pour les néologismes littéraires et ceux des humoristes. Ils remplissent souvent d'autres fonctions en sus de leur fonction ludique (accroche, connivence, arme de combat, argument de vente, provocation...) et leur durée de vie peut être variable selon les cas.

Mots clés : néologie, néologisme, matrices lexicogéniques, mot-valisation, paronymie, faussecoupe, flexion, affixation, composition, hybride, situation d'énonciation, fonctions pragmatiques, hapax conversationnel, néologismes littéraires, créations d'humoristes

\section{Néologismes ludiques : études morphologique et énonciativo-pragmatique}

\section{Introduction}

La nomination de nouveaux objets et concepts techniques et scientifiques est traditionnellement et fréquemment avancée comme explication, voire justification, des néologismes ${ }^{1}$. Mais ceux qu'on relève dans la langue courante, écrite ou orale, ne correspondent que très partiellement à ce cas. Bien d'autres raisons sont reconnaissables dans l'émergence des néologismes et parmi celles-ci la fonction ludique n'est pas la moindre. Au sein de ces néologismes ludiques on peut encore reconnaître des raisons autres que la simple recherche du plaisir du jeu de mots ou jeu avec les mots. Ils peuvent être mis à contribution pour convaincre, créer une connivence, séduire, se moquer de quelqu'un ou quelque chose, etc., et cela en lien avec la diversité des situations énonciatives. Les uns sont émis dans des conversations, d'autres dans des slogans publicitaires, d'autres encore dans des déclarations, d'hommes politiques mais pas seulement, etc. Mais on ne peut pas apparier selon des relations bi-univoques i) les différentes fonctions que jouent ces néologismes ludiques, ii) les situations énonciatives et iii) les procédés de formation (les matrices qui servent à les produire). La présentation des données nécessiterait trois dimensions (ou quatre, si l'on prend aussi en

\footnotetext{
${ }^{1}$ Cette justification figure dans les manuels de lexicologie, de terminologie et dans de nombreux articles relatifs à la néologie. C'est la seule qui soit acceptée par les puristes de toutes les époques. Sur ce point, voir Sablayrolles 2000, ch. 1 .
} 
compte la durée de vie de ces néologismes ludiques et leur diffusion, voire plus si on ajoutait d'autres paramètres) pour faire aller ensemble ces différents aspects, tous pertinents, des néologismes perçus comme ludiques. Nous prendrons pour point de départ de l'exposé leurs procédés de fabrication en nous interrogeant sur l'origine du caractère ludique qu'ont certains néologismes alors que d'autres, fabriqués apparemment de la même manière, ne l'ont pas. Quelques remarques synthétiques suivront sur des approches autres que morphologiques de la néologie ludique : diversité des situations énonciatives, diversité des fonctions autres que ludiques associées à ces néologismes, différences de leur durée de vie et de leur diffusion.

\section{Procédés de formation des néologismes ludiques}

Les travaux sur la néologie prennent prioritairement en compte l'identification de la matrice qui produit tel ou tel néologisme (v. Sablayrolles 1993 et 2000 ch. 2). Même si peu de travaux leur ont, à notre connaissance, été exclusivement consacrés ${ }^{2}$, les néologismes ludiques ont d'autant moins de raison d'échapper à cette approche que les récepteurs peuvent s'interroger sur ce qui crée l'aspect ludique. Pour les matrices mises en œuvre, la place de celles qui relèvent de la morphologie extragrammaticale est importante et, pour la morphologie constructionnelle, on relève des écarts de plusieurs types.

\subsection{Part importante de la morphologie extragrammaticale}

Relèvent de la néologie extragrammaticale ${ }^{3}$ des néologismes non produits par les mécanismes réguliers de la dérivation et de la composition. On peut en distinguer de plusieurs types.

\subsubsection{Mots-valises}

En première place viennent sans doute les mots-valises, qui sont un type de composés qui amalgament plusieurs unités lexicales en une seule avec la mise en commun d'un segment homophone. Analysés parfois comme des monstres de langue, à l'instar d'Almuth Grésillon (1985), ces assemblages ne passent pas inaperçus et les associations inattendues auxquelles procède la mot-valisation créent souvent de plaisantes chimères qui ont des « harmonies de rêve » (Laforgue $\left.{ }^{4}\right)$. L'effet de surprise, le raccourci (la condensation), la négation partielle de

\footnotetext{
${ }^{2}$ Dans son livre Les jeux de langage, Laure Hesbois traite de quelques néologismes. Pierre Guiraud, dans Les jeux de mots, emploie le mot une fois, p. 21, en renvoyant à la p. 66 où sont traités les motsvalises, avec un historique de la notion et du mot et quelques exemples, sans employer le mot néologisme. Dans la fonction 'poiétique', p. 87-88, il parle de création de mots, sans s'y appesantir. Les dictionnaires fantaisistes proposent des néologismes ludiques sans toutefois offrir de longues réflexions à leur sujet. Les travaux de Camille Vorger (2011 a et b) sur le slam traitent à l'occasion de néologismes ludiques et y consacrent une réflexion intéressante. Dans les mots d'esprit étudiés par Freud, figurent des néologismes.

${ }^{3}$ Pour des précisions sur la morphologie extragrammaticale, qui, pour faire vite, traite de ce qui n'est pas produit pas des règles, voir Fradin 2003. L'imprédicitibilté concerne tant l'aspect formel que l'aspect sémantique : le sens de ces créations n'est pas réglé, comme il l'est dans la morphologie grammaticale, où le sens des lexies est compositionnel, calculable à partir des éléments constitutifs mis en jeu dans leur fabrication.

${ }^{4}$ Lettres à un ami 64-65, cité par A. Holmes p. 34-35: «... accouplements de mots qui n'ont qu'une harmonie de rêve mais font dans la réalité des couples impossibles (et qui ont pour moi le charme insoluble, obsédant, entêtant des antinomies en métaphysique...) ».
} 
l'arbitraire du signe qui fait se rassembler des mots dont des parties du signifiant phonique ${ }^{5}$ se ressemblent nécessitent un travail particulier de fabrication et d'interprétation dont la réussite procure le plaisir particulier de la difficulté vaincue ${ }^{6}$. La présentation radicalement nouvelle ou détournée de réalités ou la création d'objets fantastiques suscitent la perception d'un décalage par rapport à l'ordinaire. Ce décalage et le caractère souvent irréel de l'objet dénommé peuvent créer l'amusement, comme le font la quasi-totalité des mots-valises des dictionnaires fantaisistes tels que ceux d'Alain Créhange (dont le titre en comporte deux savoureux : Le pornythorinque est un salopare), de Finkielkraut, de Galisson, etc.

Dans une proposition de traduction des Nuées d'Aristophane, un des personnages de la comédie est gêné dans ses mouvements par le vêtement dans lequel il est enveloppé : il est emmaillobêté (embêté par son maillot). La condamnation de la corruption des classes dirigeantes se fait sous un mode humoristique dans la création de ripoublique (République des ripoux) par Jean-Marie Le Pen, leader de l'extrême droite nationaliste française.

Certains mots-valises mettent en jeu un ou deux noms propres comme maastricheur (où Chevènement qualifie de tricheur le traité de Maastricht), ou sarkollandisation qui nie la différence des deux leaders politiques que sont Sarko[zy] et Hollande et condamne l'importance accordée à ces deux candidats à la Présidence de la République française au détriment d'autres faisant des propositions plus radicales. Les homophonies sont plus lâches mais pas inexistantes dans le croisement des deux noms propres composés Giscard d'Estaing et Chaban-Delmas, pour affirmer leur interchangeabilité : Chaban d'Estaing et Giscard

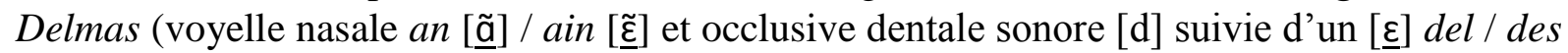
avec aussi un parallélisme de structure : deux mots de deux syllabes), candidats rivaux aux élections présidentielles de 1974.

Un slogan publicitaire encore utilisé par l'enseigne Castorama mêle son nom et l'adjectif familier fastoche «facile » avec le segment graphique commun -asto- à première syllabe homophone et à seconde syllabe très proche phonétiquement ${ }^{7}$ avec seulement une différence d'aperture d'un degré de la lettre $o$ [asto]/ [asto] pour former castoche ; ce qui signifie que le bricolage est fastoche avec Castorama.

Sans doute peut-on parler d'expressions-valises (cf. Camille Vorger 2011) dans le cas d'amalgame de deux mots-composés ou expressions de base comme serial menteur issu de la superposition de supermenteur (marionnette de Chirac de l'émission télévisée satirique Les Guignols) et du schéma serial X-eur. C'est ainsi que Le Pen exprimait son aversion pour Jacques Chirac. Sa maîtrise de la langue française exclut de considérer comme de simples

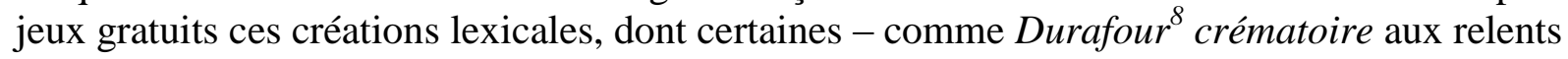
antisémites - lui ont valu des condamnations en justice.

Parfois des mots-valises se fondent de surcroît sur de fausses analyses volontaires. Ainsi pyrowoman «femme qui aime faire du feu dans la cheminée » ou self made mélomane « homme qui ne doit sa culture musicale qu'à lui-même et pas par héritage », dans lesquels le man anglais «homme » est confondu avec mane qui marque l'attrait pour quelque chose, parfois poussé à l'excès jusqu'à la folie. Un degré supplémentaire de déni de l'arbitraire du signe est sans doute atteint dans de tels rapprochements de segments homophones relevant de

\footnotetext{
${ }^{5}$ La graphie, souvent identique, ne l'est pas nécessairement comme dans sangsuel croisant sang et sensuel dans une Complainte de Laforgue.

${ }^{6}$ Ceci vaut pour tous les mots-valises, et pas seulement pour les mots-valises ludiques, mais cette caractéristique est également présente dans les jeux de mots en général, et même dans nombre de blagues : ne dit-on pas qu'il faut trois personnes pour qu'une blague soit réussie, celui qui la raconte, un auditeur qui la comprend et un autre qui ne la comprend pas ?

${ }^{7}$ Dans les mots-valises, les homophonies peuvent parfois être imparfaites dans la partie commune comme dans élevache, élevage de vaches cité par Fradin (2000).

${ }^{8}$ Homme politique de centre droit.
} 
codes linguistiques distincts. Il y a, en tout cas, une transgression supplémentaire dans la création d'hybrides, mélangeant des langues dans une même unité lexicale. C'est aussi le cas de Waterbraguette (Libération) à propos du Monicagate (scandale des frasques sexuelles de Bill Clinton avec une stagiaire nommée Monica Lewinsky ${ }^{9}$ ).

Par ailleurs si tous les mots-valises ne sont pas nécessairement ludiques ${ }^{10}$, ces fusions de mots sur la base d'une ressemblance phonique due au hasard jouent fondamentalement avec le signifiant pour créer, parfois secondairement, des signifiés nouveaux, souvent bizarres et étonnants. Ce n'est sans doute pas par hasard que nombre de dictionnaires fantaisistes, recueils ludiques de mots qui n'existent pas, tels ceux d'Alain Finkielkraut (Ralentir motsvalises, 1979), de Jean-Loup Chiflet (Le cafard laqué. Les mots-portemanteaux, 1999 ou Mais que fait l'Académie? Le dictionnaire des mots qui devraient exister, 2002), d'Alain Créhange (Le pornithorynque est un salopare, 2004), etc. soient constitués (quasi)exclusivement de mots-valises ${ }^{11}$.

\subsubsection{Déformations paronymiques, fausses coupes}

Relèvent également de la morphologie extragrammaticale des déformations paronymiques (avec des étymologies dites «populaires» par pseudomotivation, et des remotivations) et des fausses coupes (par agglutination et déglutination). Si ces procédés ont joué un rôle, non ludique, dans l'évolution du français ${ }^{12}$, on relève des créations parodiques de ce type dans divers types d'énoncés, en particulier dans la bande dessinée, dans la bouche d'humoristes ou sous la plume d'auteurs comme Frédéric Dard dans les romans de San Antonio, etc. pour caricaturer des manières de parler populaires fautives.

Un auteur de bande dessinée (dans Hara-Kiri du 10 mars 1993) met ainsi dans la bouche de ses personnages des fausses coupes et approximations phoniques de type paronymique : la nesthésie, la bulance, avec une déformation encore plus profonde dans un cactus dans le myocarde ${ }^{13}$. Le décalage entre les fautes de prononciation des personnages et

\footnotetext{
${ }^{9}$ Première attestation, à notre connaissance, le $1^{\text {er }}$ septembre 1998 et une requête sur Google le 19 novembre 2012 indique 195 pages pour ce mot-valise.

${ }^{10}$ Notons que des procédés proches de la mot-valisation, et parfois confondus avec elle, la compocation (composé de deux mots tronqués comme héliport alliant aéroport et hélicoptère) et la fractocomposition (un des deux éléments du composé est représenté par une partie de lui-même appelée fractomorphème ou mieux fractolexème comme téléspectateur où télé vaut pour télévision) n'ont que rarement cette propriété, étant le plus souvent purement dénominatifs dans des domaines de spécialité du moins à l'origine. Cette possibilité d'être ludique, le caractère non réglé du sens (voir Fradin 2000, qui montre que les rapports entre les éléments du mot-valise peuvent être très divers et que le contexte est déterminant pour l'interprétation) ainsi que le fait de comporter un ou plusieurs segments homophones qui, provoquant la fusion, conduisent à distinguer nettement la mot-valisation de la compocation et de la fractocomposition, ce que ne fait pas une approche scalaire de l'amalgame, comme celle défendue par Renner (v. le volume en anglais). D'autres articles traitent de mots-valises ludiques, comme celles de Kabatek, Knospe, etc. Si la mot-valisation est ancienne (sorbonagre, croisant Sorbonne et onagre "âne », sous la plume de Rabelais), la fractocomposition et surtout la compocation, dont on doit le concept à Fabienne Cusin-Berche (2003), sont d'apparition et de développement beaucoup plus récents.

${ }_{11}$ À ce sujet, voir Arnaud Léturgie (2011) et sa thèse (2012).

${ }^{12}$ Comme l'agglutination de l'article avec le nom dans l'uette $=>$ la luette, ou la réinterprétation de fame «renommée » disparue en femme homophone hétérographe : remède de bonne fame => femme, etc.

${ }^{13} \mathrm{Au}$ lieu de l'infarctus qu'on entend d'ailleurs de plus en plus souvent prononcé infractus comme si quelque chose était cassé. La fracture du myocarde est le titre d'un film ainsi dénommé par reprise de
} 
la correction orthographique, due à l'auteur de la bande dessinée, avec l'emploi des graphèmes - $y$ - et -th- conformes à l'étymologie grecque (et non $i$ et $t:$ nestésie, miocarde) est une source supplémentaire de comique et range les lecteurs du côté de l'auteur dans leur supériorité moqueuse en face de personnages fictifs grossiers et incultes.

L'humoriste québécois Marc Favreau dit Sol, dans le recueil intitulé de la jolie expression-valise Je m'égalomane à moi-même (croisant égal à moi-même et mégalomane «Je continue d'être mégalomane comme je l'étais »), incarne un personnage de naïf qui s'extasie devant l'Altesse de l'air lors de son premier voyage en avion. La synapsie hôtesse de l'air est mal encodée par le personnage du sketch, qui, ébloui par l'hexis et la beauté de la jeune femme, la considère comme une personne de haut statut social, une altesse. L'hypothèse de la création de cette synapsie par mot-valisation croisant altesse et hôtesse de l'air avec le segment homophone [t $\underline{\varepsilon}$ ] est exclue en contexte, du fait de la naïveté du personnage. Le même humoriste, cité par Laure Hesbois (1988), évoque, avec une fausse coupe, Les Gyptiens qui font remarquablement des pyramides humaines dans un spectacle de cirque. La suite phonique [leGipsjã ], l'Égyptien, est analysée par le personnage du sketch comme un mot pluriel contenant l'article défini les: les Gyptiens.

Le personnage de Bérurier, entre autres, dans les romans de San Antonio écorche les mots et expressions pour le plus grand plaisir des lecteurs. Dans ma ford intérieure vaut pour dans mon for intérieur. Le mot graphique for, rare et usité seulement dans l'expression dans mon/ton/son ... for intérieur ( « en moi-même, toi-même, etc. ») est rapproché d'un mot voisin, plus fréquent dans la langue courante, le nom d'une marque automobile, Ford. Ce rapprochement se fait d'autant plus facilement qu'existe la catégorie de véhicules 'conduite intérieure' qui s'opposait aux véhicules dont le chauffeur était en dehors de l'habitacle, et qui se sont appelées autrefois des limousines ${ }^{14}$. Il y a aussi des déformations progressives comme

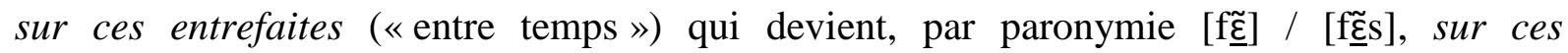
entrefesses, puis quelques chapitres plus loin encore, en utilisant un synonyme de fesses, sur ces entremiches.

Une publicité pour l'eau de Javel joue sur la paronymie javel / je vais le dans [zavễl] « Javel dire à tout le monde ».

Des fautes involontaires - et parfois répétées -s de locuteurs lambda amusent ceux qui les repèrent, même si aucune intention ludique ne présidait à leur émission. C'est le cas du mec plus ultra au lieu du nec plus ultra ou des pastilles Lajoignie au lieu de Lajaunie par substitution du nom d'un homme politique connu à l'époque ${ }^{15}$. Pour un non latiniste nec n'est pas identifiable (c'est une conjonction de coordination négative «et ne...pas »), à la différence de plus et ultra qui existent dans la langue française. Selon le procédé traditionnel qui consiste à ramener de l'inconnu au connu le plus proche, la nasale dentale devient labiale

la dénomination fautive qu'emploient les enfants de la fiction, après le décès brutal de la mère de l'un d'entre eux.

${ }^{14}$ D'après Wikipedia se référant à l'émission «Merci professeur» de Bernard Cerquiglini : «Au départ, la limousine est un type de véhicule hippomobile en usage dans la province française du Limousin. Son nom pourrait venir d'une vaste pèlerine, la limousine, que les habitants du Limousin portaient autrefois pour se protéger de la pluie ; laquelle aurait donné son nom aux premières voitures nommées limousines où seules les places arrière étaient protégées par des vitres. »

${ }^{15}$ Confusion commise dans une pharmacie et racontée, dans une file d'attente où je me trouvais, par une jeune étudiante à ses condisciples hilares, sans qu'on sache s'il s'agissait originellement d'une véritable erreur ou d'un simple lapsus. Les conditions d'énonciation du propos rapporté excluent une faute volontaire (on n'emploie pas un mot impropre volontairement dans la commande d'un médicament. C'est seulement dans des échanges entre pairs qu'on peut procéder ainsi). En tout état de cause, aussi bien la narratrice que ses interlocuteurs comprenaient la méprise et s'en amusaient. 
pour former le mot mec («quelqu'un »), familier et fréquent. On dénomme ainsi quelqu'un de remarquable.

Dans tous ces cas, c'est le décalage entre les connaissances linguistiques des récepteurs et les productions fautives, réelles ou supposées, de personnes incultes ou naïves qui provoque l'amusement voire l'hilarité : on aime à se moquer des faiblesses des autres. Les fautes feintes permettent des transgressions du code et il peut y avoir du plaisir dans la transgression mais ces transgressions linguistiques sont mimées et pas véritables, à la différence des véritables fautes. Elles créent des connivences entre l'émetteur et son / ses récepteurs interprétants ${ }^{16}$ sur le dos d'un tiers (absent mais dont on imagine qu'il ferait de telles fautes) ou d'un être de papier.

\subsubsection{Matrices phraséologiques : créations et détournements d'expressions}

Les détournements d'expression que Camille Vorger a adoptés dans sa thèse $(2011)^{17}$ en rebaptisant la matrice du nom de matrice phraséologique ne sont pas unanimement reconnus comme relevant de la néologie ${ }^{18}$, mais si on considère qu'il y a dans ces cas des innovations qui se manifestent au niveau d'unités lexicales, cela relève bien de la néologie, même si ce n'est pas la néologie prototypique, et cette matrice relève également de la morphologie extragrammaticale. Le point crucial est que le mot composé ou l'expression néologiques ne sont interprétables que par l'identification de la lexie originelle qui n'apparaît pas en entier.

Ainsi les synapsies ${ }^{19}$ brèves de wagon ou brèves de pouvoir ne sont interprétables qu'à partir du modèle de brèves de comptoir, titre d'un livre qui rapporte les propos brefs et souvent à l'emporte-pièce entendus aux comptoirs des bars. Ce type de création est ponctuel et analogique, même s'il peut donner naissance à une petite série d'expressions faites sur le même moule. La reconnaissance de la formule d'origine et le travail d'interprétation spécifique exigé par la modification apportée procurent du plaisir à celui qui réussit cette épreuve. Comme par ailleurs ces brèves de comptoir sont souvent humoristiques, les propos échangés par des banlieusards qui se retrouvent chaque jour dans les mêmes trains - les brèves de wagon - sont du même type. Il n'en va guère autrement pour les brèves de pouvoir que sont certains propos tenus par d'anciens premiers ministres sur leur expérience à Matignon, puisque ces propos sont essentiellement des anecdotes plaisantes de mésaventures vécues par eux dans l'exercice de leurs fonctions.

Le mot composé serial papa entre dans une série en serial X-eur-sans le -eur attendu et cette attente déçue ainsi que le décalage de registre entre les deux formants - un mot

16 Sur ce concept d'interprétant, voir Grunig 1985. L'emploi d'interprétant de préférence à allocutaire, interlocuteur, etc. met l'accent sur les nombreuses tâches, diverses, et étudiées une à une, auxquelles se livre le récepteur d'un dire, d'un énoncé (qu'il en soit le destinataire ou non) pour en construire le sens, avec les inévitables distorsions entre le sens émis par le locuteur et le sens construit par l'interprétant : c'est la fuite du sens à droite.

${ }^{17}$ Poétique du slam: de la scène à l'école (Néologie, néostyles et créativité lexicale), dirigée par Francis Grossmann et Dominique Abry, soutenue le 23 novembre 2011 à l'Université de Grenoble.

${ }^{18}$ Certains n'y voient qu'un défigement, processus appliqué à une séquence figée qui la modifie, mais il s'agit bien d'une innovation au niveau d'une unité lexicale et cela relève donc de la néologie, au moins à un niveau périphérique. Par ailleurs parler de défigement ne dit rien des conséquences sémantiques éventuelles. Il faut en effet faire le départ entre des modifications "gratuites », qui ne sont que des clins d'œil (fréquentes dans les titres de la presse) et des modifications créatrices de sens, dans lesquelles l'interprétant doit reconnaître la séquence figée originelle et combiner son sens avec le sens de l'élément nouveau.

${ }^{19}$ C'est ainsi que Benveniste (1974) a nommé les composés dont les éléments sont reliés par un joncteur et auxquels il a voulu donner une dénomination propre du fait de leur grande expansion. 
d'origine anglaise et un mot familier français - ont un incontestable pouvoir comique, même si le mot dénomme une réalité qui l'est moins : un homme jeune géniteur, avec de multiples conquêtes féminines d'un soir, de plusieurs dizaines d'enfants, dont il ne s'occupe pas.

L'expression être les dindons de la farce («se faire avoir, être la dupe dans une affaire ») a été détournée dans un tract, distribué par des étudiants à la sortie de l'Université Paris 13 en 2010 ou 2011, qui exprimaient leur colère en déclarant, avec un jeu de mots, ne pas vouloir être les dindons de la crise, c'est-à-dire être les victimes de la crise économique dont ils ne sont pas responsables.

Des détournements d'expression se manifestent aussi dans le fait de donner à une expression une lecture compositionnelle au lieu de sa signification conventionnelle, souvent opaque. Ainsi sortir son couteau pour un oui ou pour un non indique une activité agressive, voire criminelle, gratuite de surcroît, et on a pu s'étonner de lire dans un slogan publicitaire pour le scoutisme ; "Chez les Scouts de France, on sort son couteau pour un oui ou pour un non »: il ne s'agissait alors que de l'apprentissage de la débrouillardise et du travail manuel par le recours aux moyens du bord (le simple canif qu'un scout digne de ce nom porte toujours sur lui) à défaut d'une trousse à outils bien fournie. Il s'agit de la multiplicité des situations où un couteau peut être utilisé en dehors de sa fonction par destination, qui est celle de couper (servir de tournevis, de levier, de grattoir, etc.). Une voiture qui dépasse les bornes $^{20}$ a simplement une grande autonomie et ne manifeste aucun excès comme le sens conventionnel de l'expression figée le laisserait attendre.

Des créations d'expressions, souvent métaphoriques, relèvent de la néologie ludique du fait même du transfert entre des domaines étrangers l'un à l'autre. On plaque ainsi sur de l'humain ce qui relevait de réalités matérielles : la lenteur d'esprit est assimilée au bas débit dans le domaine informatique : ne pas faire du huit mégabits, ne pas être branché haut débit, ou bien est comparée à un bâtiment qui n'a pas la lumière à tous les étages.

Ces matrices phraséologiques ont souvent une fonction colludique, pour reprendre le terme de Camille Vorger (2011a et b) : elles créent une connivence par un savoir partagé (les expressions figées d'origine dans le cas des détournements et le sens propre dans le cas des emplois figurés) et cette connivence est encore augmentée par le plaisir procuré et reçu du jeu avec les mots.

\subsubsection{Néologie flexionnelle}

Encore plus périphérique dans une conception scalaire de la néologie, dans la mesure où il y a innovation au niveau d'une unité lexicale sans création d'une nouvelle unité lexicale, la néologie flexionnelle ressortit en fait au système de la langue, et donc au grammatical. Sa place est donc ambiguë et c'est par défaut qu'on la présente ici à la charnière de la partie extragrammaticale et de la morphologie constructionnelle. La maîtrise d'une lexie comprend en effet non seulement la connaissance de son signifiant (graphique et phonique), de son signifié (son ou ses acceptions), mais encore sa flexion (classe paradigmatique de rattachement pour les mots fléchis et particularités flexionnelles éventuelles : formes défectives, formes doubles concurrentes, faits d'allomorphie ou de supplétisme, etc. $)^{21}$. Du coup toute innovation dans la flexion relève de la néologie, même si ce n'est pas de la néologie prototypique comme un nouveau dérivé ou un emploi figuré.

\footnotetext{
${ }^{20}$ Dans une page publicitaire (septembre 2001) pour un modèle économe en carburant, sur fond du jeu des mille bornes.

${ }^{21}$ Elle comprend aussi sa combinatoire, i.e. sa construction syntaxique et son schéma argumental formulé en termes de classes d'objets comme dans le modèle de Gaston Gross (2012 entre autres). Une innovation combinatoire relève aussi de la néologie et peut être ludique, voir infra.
} 
À côté de fautes de production du type vous en buverez encore ou je tremblera qui ne sont guère plaisantes que quand on les raconte à autrui, on observe des créations de formes flexionnelles inusitées dont certaines - mais pas toutes - sont ludiques.

La petite fille qui réplique à son père déclarant qu'ils étaient serrés comme des sardines que lui est un sardin s'amuse à donner un masculin au mot sardine qui n'existe qu'au féminin, en tenant compte du sexe masculin de son père. Il ne s'agit pas d'ignorance mais de l'application délibérée du système morphologique du français d'opposition des genres masculin et féminin en ôtant le $-e$ caduc final de féminin avec son incidence sur la prononciation de la syllabe finale, parallèlement à naine / nain, crétine / crétin, etc. L'extension analogique indue provoque le sourire et la connivence, par la conscience partagée d'une faute volontaire: je sais que je me trompe et je sais que tu sais que je me trompe délibérément, pour rire.

L'humoriste Alphonse Allais ${ }^{22}$ joue avec la catégorie du nombre en employant mon émolument au singulier. Quand on lui fait remarquer qu'on dit mes émoluments, il réplique qu'il ne veut pas déranger le pluriel pour si peu, faisant comme si l'opposition singulier / pluriel reflétait l'importance de la somme en question. Tout est dans le 'comme si', qui consiste à appliquer une sorte de logique mentale associant pluralité et importance vs singulier et petitesse dans le fonctionnement d'une langue naturelle, qui n'obéit pas à ces principes. C'est une manière élégante et drôle de se plaindre de la faiblesse de sa rémunération.

Ces inventions flexionnelles peuvent avoir un aspect contestataire à côté de l'aspect ludique qui retient l'attention. Ainsi des futurs nous nous en allerons et je repartira du chanteur Renaud («dès que le vent soufflera », album Morgane de toi, 1983), ou, plus anciennement encore d'un slogan politique (du Parti Communiste ou du syndicat CGT, Confédération Générale du travail) qui invente un nouveau paradigme, présenté sur une affiche comme un tableau de conjugaison de manuel scolaire, au verbe travailler avec une $3^{\mathrm{e}}$ personne du pluriel inattendue :
je travaille,
tu travailles
il travaille
nous travaillons
vous travaillez
ils profitent

La surprise provoquée par la dernière forme du paradigme ne peut pas manquer d'arracher un sourire, même à des capitalistes forcenés, peu suspects de sympathie pour les auteurs de ce slogan. Il s'agit en fait de constituer une apparente conjugaison supplétive, en faisant appel à plusieurs radicaux, mais la forme de $3{ }^{\text {ème }}$ personne du pluriel existe indépendamment, c'est celle du verbe profiter. Son surgissement, inattendu, après cinq formes régulières, constitue la pointe finale du slogan.

Certaines fautes feintes se sont ancrées en langue (et ne sont donc plus néologiques) du type je m'a gouré («je me suis trompé ») qui cumule les infractions (lexie familière gourer, auxiliaire avoir et non être attendu pour ce verbe pronominal, et faute de conjugaison avec $a$ au lieu de $a i$ à la $1^{\text {ère }}$ personne du singulier). Son emploi est là encore colludique, du

\footnotetext{
${ }^{22}$ Une vérification sur internet montre que l'anecdote est rapportée fréquemment, mais sans référence précise à l'intérieur de l'œuvre d'Alphonse Allais. Elle est une fois attribuée à Labiche, sans doute par erreur, et on trouve aussi un certain nombre d'emplois de ce mot au singulier sans volonté de transgression ludique.
} 
fait de la conscience partagée de ces transgressions et des décalages par rapport à la forme conventionnelle attendue. Il s'agit de fautes mimées dont personne n'est dupe, ni l'utilisateur ni ses allocutaires.

\section{2. Écarts de divers types dans la morphologie constructionnelle}

La plupart des mots possibles non attestés et qui à un moment précis le deviennent, par néologie, n'ont aucun caractère ludique en soi $^{23}$. Beaucoup passent même d'ailleurs inaperçus comme le indécorable de Saussure ou feuilleteur, fienteux créés par Laforgue dans deux de ses Complaintes ${ }^{24}$. L'aspect ludique vient de l'aspect inattendu, paradoxal de l'association des formants des néologismes, d'un point de vue sémantique ou morphologique.

\subsubsection{Association inattendue d'éléments d'un point de vue sémantique}

Un effet de surprise peut être recherché avec le surgissement de signifiés paradoxaux ou contradictoires qui peut de surcroît amuser du fait de son inventivité. Cela se manifeste dans différents types de cas distingués seulement pour la clarté de l'exposé. Le mécanisme est le même.

\subsubsection{Dans des composés 'réguliers'}

Boris Vian a créé quelques objets étonnants comme le ratatine-ordure dans sa chanson «La Complainte des arts ménagers » ou le lance-hostie dans L'Automne à Pékin. L'aspect familier de ratatiner ne prédispose pas ce verbe à être utilisé comme membre de composé, dans la dénomination d'un objet ménager qui servirait à compacter les ordures comme dans les camions-bennes qui tassent et compressent le contenu des poubelles qui y est déversé. L'invention d'un tel objet à usage domestique paraît par ailleurs peu probable (et rien de tel n'a de fait été inventé depuis la création du mot par Boris Vian dans les années 50).

La transgression est plus violente dans le cas du lance-hostie puisqu'elle relève du sacrilège : l'hostie étant une galette de pain azyme utilisée dans la liturgie chrétienne comme étant ou représentant le corps du Christ. Elle est assimilée à un projectile comme une grenade, un missile... (lance-grenade, lance-missile, etc.). Le décalage entre l'aspect religieux et l'aspect militaire produit, sauf chez ceux que cela offusque dans leur foi, un aspect comique

\footnotetext{
${ }^{23}$ Sur 2500 néologismes entrés depuis 2009 dans la base Neologia du laboratoire LDI UMR 7187, il n'y en a qu'une minorité à laquelle on pourrait assigner une fonction ludique, mais il est délicat de trancher à tous les coups. La dimension ludique n'est pas prise en compte dans la grille d'analyse (qui compte plus d'une trentaine d'informations), pour des raisons explicitées dans Sablayrolles 2000 (partie III, ch. 9) : les fonctions assignables aux néologismes sont trop nombreuses, mêlées, variables selon les interprétants, et parfois indécidables, pour qu'elles soient systématiquement indiquées dans une grille d'analyse. Rappelons aussi que la doxa dit que la raison essentielle de l'apparition des néologismes est la dénomination de nouvelles découvertes ou de nouveaux objets. Sont ainsi fréquemment opposés des néologismes dénominatifs et des néologismes de luxe, stylistiques. Cette dichotomie simpliste rend mal compte de la multiplicité des fonctions des néologismes, qui ont, de surcroît, toujours une fonction dénominative, et qui ne sont jamais de luxe, gratuits pour celui que les crée, comme Matoré (1952) l'avait montré.

${ }^{24}$ Mais l'analogie a des caprices disait Saussure; ce qui explique le sentiment de bizarrerie ressenti devant frêleur, frêlité, frêlesse ou frêlure malgré l'existence de couples très semblables ayant comme bases des adjectifs monosyllabiques : pâle: pâleur $=$ frêle $=>$ ?frêleur ou rude: rudesse $=$ frêle $\Rightarrow$ ?frêlesse ou encore cher: cherté ou sourd: surdité $=$ frêle $=>$ ?frêletélfrêlité ou enfin froid: froidure $=$ frêle $=>$ ?frêlure. (Sur ce point voir Sablayrolles à paraître).
} 
du fait du caractère saugrenu de cet instrument inventé par Boris Vian (L'Automne à Pékin, 1947).

\subsubsection{Dans des synapsies}

À côté du ratatine-ordure le même Boris Vian a créé, dans la même chanson, le canon à patates, synapsie dans laquelle les pommes de terre, sous la dénomination familière patate, deviennent des projectiles, comme les hosties de l'exemple précédent. Un canon est une arme qui envoie des projectiles (boulet, obus...) et pas des végétaux et / ou aliments comme des patates. La confusion des ustensiles ménagers (les différents types de robots, mixeurs, etc.) qui se développaient pendant les trente glorieuses, période où a été écrit ce texte, et d'un type d'armes est source de comique.

Pour rester dans le domaine militaire, Jean-Luc Mélenchon a traité le candidat François Hollande de capitaine de pédalo pendant la dernière campagne présidentielle. Capitaine est un grade militaire, d'officiers supérieurs dans la marine (capitaines de corvette, de frégate et de vaisseau), et l'objet de loisir inoffensif qu'est un pédalo qui ne vogue que sur des eaux calmes est inattendu dans la série des grades et dévalorise celui qui est qualifié ainsi : au lieu d'être un chef de guerre avec des pouvoirs et des compétences, Hollande est présenté comme un simple touriste ou vacancier incapable de s'attaquer aux problèmes politiques, économiques que doit traiter un président de la République (qui est aussi en France le chef des armées). La rupture dans la série des bâtiments de guerre (corvette, vaisseau, frégate...) avec l'inclusion d'une petite embarcation de loisir est source de comique.

\subsubsection{Dans des dérivés}

L'association d'un suffixe savant du domaine de la médecine (-ite) et d'un nom dénommant un objet prosaïque (biberon), pris en outre dans un sens second familier ${ }^{25}$ crée également une rupture comique dans biberonite. Le suffixe -ite a en effet pris le sens de « inflammation» dans le domaine médical pour des noms de pathologies (appendicite, trachéite... $)^{26}$. Le décalage entre le formant savant et l'acception familière de biberon(ner) pour «boire / être alcoolique » crée un contraste qui provoque le rire d'autant plus que, dans certaines situations, l'allusion à l'excès de boissons alcoolisées est source de comique ${ }^{27}$. La création de ce néologisme par Debidour dans sa traduction des Guêpes d'Aristophane est un faux euphémisme (on laisse entendre une pathologie sans la nommer, mais il ne s'agit pas, dans ce contexte comique, d'une véritable atténuation; bien au contraire) que les lecteurs ou spectateurs décryptent sans difficulté et ils apprécient cette manière détournée d'évoquer une réalité à laquelle elle donne un aspect humoristique.

L'omniprésence du mensonge, tant dans la sphère publique (en particulier politique) que dans la sphère privée, a conduit un journaliste de l'hebdomadaire Marianne $\left(\mathrm{n}^{\circ}\right.$ du 27 juillet 2013) à créer le terme mensongite, comme s'il s'agissait d'une maladie chronique. L'assimilation d'un comportement personnel (présenté traditionnellement comme un défaut) à une sorte de maladie contagieuse qui affecte tout le corps social provoque un sourire, du fait du décalage des domaines (morale/ santé) et de l'hyperbole (avec une omniprésence du

\footnotetext{
${ }^{25} \mathrm{Ce}$ sens second est en fait présent dans le verbe biberonner qui signifie «boire beaucoup, être alcoolique $»$.

${ }^{26}$ Un des relecteurs, que je remercie de sa lecture vigilante, indique des équivalents allemands (Telephonitis "usage immodéré du téléphone », Apostrophitis "usage immodéré des apostrophes », etc. ainsi que l'existence d'études allemandes et espagnoles sur ce suffixe -itis.

${ }^{27}$ Comme le montre le célèbre sketch de Bourvil, «L'eau ferrugineuse ».
} 
mensonge qui infecterait toute la vie publique comme un virus qui se répandrait, ce qui est exagéré).

\subsubsection{Cas de remotivation}

Le jeu entre un sens conventionnel opaque d'un mot complexe ou d'une expression et la lecture compositionnelle est une source inépuisable de comique. Raymond Devos a eu recours, dans son sketch La mer, à ce mécanisme avec les mots préfixés, non néologiques il est vrai, démonter et remonter:

- Je voudrais voir la mer.

- Vous ne pouvez pas la voir, la mer, elle est démontée.

- Vous la remontez quand? (Devos)

L'erreur du personnage incarné par Raymond Devos est de prendre remonté comme antonyme de démonté et non de calme qui s'impose quand il s'agit de la $\operatorname{mer}^{28}$. On note une infraction au schéma argumental de ces deux verbes ou adjectifs prédicatifs en termes de classes d'objets ${ }^{29}$.

Dans un tout autre contexte, on peut imaginer la réaction du serveur à qui un étranger désirant un jus de pomme demanderait une pommade, sur le modèle de orangeade, citronnade, etc. Cet exemple est emprunté aux travaux de Corbin (1987) qui montre que, même lorsqu'un mot a un sens qui ne correspond pas à celui attendu par l'application des règles de construction des mots ( $\mathrm{RCM})$ qui associent des opérations morphologiques et sémantiques (c'est un modèle dit, pour cette raison, associatif), ce dernier sens est récupérable. La preuve en est précisément la fabrication possible de blagues ou de jeux de mots fondés sur le décalage entre le sens prévisible construit par les règles et le sens conventionnel attesté.

On aurait pu classer ici aussi l'exemple de elle dépasse les bornes avec les deux lectures conventionnelle et compositionnelle.

\subsubsection{Associations bizarres morphologiquement mais non extragrammaticales}

Parfois, c'est au niveau des signifiants qu'apparaît principalement une bizarrerie qui amuse. C'est le cas de composés hybrides ou de néologismes savants de fantaisie. Comme composés, ils relèvent des matrices grammaticales, avec des règles, mais à la différence de la

\footnotetext{
${ }^{28}$ Le participe passé démonté est antonyme de monté, puis remonté est antonyme de démonté pour quelque chose qui a été assemblé une première fois puis remis dans l'état initial de pièces détachées. Dans «la mer est démontée », démontée fonctionne comme un adjectif prédicatif, attribut du sujet la mer et non comme forme de $3^{\text {ème }}$ personne de l'indicatif présent passif du verbe démonter, forme qui correspond à l'ultime question, « Vous la remontez quand ? » où le verbe est repris à la voix active.

${ }^{29}$ Le modèle des classes d'objets (v. Denis Le Pesant et Michel Mathieu Colas (1994) et Gaston Gross (2012)) a pour objectif de décrire une langue en faisant des dictionnaires de phrases élémentaires, c'est-à-dire de prédicats (verbes, noms, adjectifs...) saturés par leurs arguments (avec la suite la plus longue de leurs arguments). Ce modèle permet de différencier les emplois selon les classes d'arguments qui leur sont associées. Ainsi y a-t-il au moins deux verbes prédicatifs démonter: démonter1, N0 <hum>, N1<hum> «faire tomber quelqu'un de sa monture »; démonter2, N0<hum>, $\mathrm{N} 1<$ objet concret manufacturé, artefact〉 « désassembler des éléments qui avaient été assemblés ». De même il y a au moins deux adjectifs prédicatifs démonté : démonté1 N0<hum> «déconcerté, troublé » et démonté2 N0 <objet naturel, mer> «agité ». Le sketch de Devos est fondé sur la confusion par le personnage de l'adjectif prédicatif démonté2 avec le verbe prédicatif démonter2.
} 
plupart des autres composés, ils ont un aspect ludique, venant de la combinaison des éléments assemblés.

\subsubsection{Composés hybrides}

L'association du mot enfantin nounours (avec réduplication de l'attaque du mot ours et fausse coupe par agglutination de la consonne - $n$ - de l'article indéfini un prononcé en liaison [nurs] pour [urs]) avec le mot d'origine grecque thérapie donne nounoursothérapie qui, sous son aspect amusant du fait de cette alliance de mots de registres différents, nomme une réalité sérieuse : la préoccupation du moral et de l'affectivité des enfants hospitalisés. Pour offrir de la détente aux personnes stressées, ont été créés des bars à chats où les clients boivent leurs consommations, entourés de chats qu'ils peuvent caresser dans ce que les journalistes de Marianne (17 août 2013) nomment du beau néologisme hybride formé d'un nom d'origine onomatopéique et du même mot savant thérapie, la ronronthérapie. Ces deux exemples seront peut-être à l'origine d'une série associant thérapie à un mot de type familier, mais cela ne semble pas encore être le cas (comme ça l'est pour serial X-eur).

\subsubsection{Néologismes savants « héroï-comiques »:}

Des gens créent pour le plaisir des composés savants pour renommer des réalités quotidiennes voire prosaïques et certains les diffusent sur leur site (par exemple: http://www.cledut.net/xylo.htm consulté régulièrement jusqu'en 2012). C'est un autre ressort du comique systématique d'activité sur la langue qui se fonde sur le décalage entre le statut des formants et les réalités dénommées.

Kératocéphale est formé du grec kéras, -atos «corne » et kephalé «tête ». Il signifie «à la tête cornue », mais la définition qui en est proposée ne renvoie pas au cerf ou autres animaux pourvus de cornes mais est le synonyme de соси (représenté traditionnellement avec des cornes et objet par ailleurs de nombreuses plaisanteries).

La xyloglosse est la «langue de bois ». C'est ce mot qui est utilisé pour dénommer le site indiqué ci-dessus (qui comporte xylo «bois » dans son adresse), pour parodier les termes techniques savants jugés envahissants. Le calque savant de la synapsie d'usage courant se présente comme une devinette pour qui ne connaît pas le grec et la révélation du sens produit un certain amusement par la reconnaissance de quelque chose de connu, sous un terme inconnu.

Mêlant les deux procédés - et placé pour cela en toute fin de 1.2.2. -, Philipe Meyer, pour se gausser de ce travers moderne de termes pédants, faisait de la surenchère en créant des mots savants ou hybrides particulièrement ridicules comme institut de légumologie appliquée («magasin de fruits et légumes »), fruitdemerologue («spécialiste des fruits de mer»), vectorologue de l'écriture («spécialiste en rédaction de textes »), vélocipédophile («amateur de vélo »), etc.

\section{Autres approches, énonciativo-pragmatiques, des néologismes ludiques}

Les procédés morphologiques mis en œuvre pour former des néologismes ludiques sont donc divers comme sont diverses les situations énonciatives où ils apparaissent ainsi que les fonctions autres que ludiques qu'ils peuvent remplir. La plupart d'entre eux sont fugaces, mais certains peuvent s'installer dans la durée.

\subsection{Types de situations énonciatives et d'énonciateurs privilégiés}


Les exemples cités dans la première partie viennent de quelques sources principales, parmi lesquelles il faut mettre au premier rang les humoristes, du moins certains d'entre eux qui fondent leurs sketchs sur l'usage de la langue. Raymond Devos est sans doute le plus connu en français. Nous avons cité aussi le Québécois Sol, mais bien d'autres pourraient l'être comme les Frères ennemis par exemple.

La littérature - au sens large, avec des genres privilégiés comme les complaintes telles celles de Laforgue, les chansons, les chroniques, les pamphlets - est un vivier de jeux de mots. Mais les genres nobles, comme la tragédie, du moins dans la tradition française, excluent la néologie et encore plus les jeux de mots : pour Victor Hugo, « le calembour est la fiente de l'esprit qui vole » (Les Misérables, t. 1, 1862). Les ambiguïtés (Il sortit de la vie comme un vieillard en sort / un vieil hareng saur) ainsi que certaines séquences (ceux-ci sont / saucisson) sont à éviter. Mais Corneille a maintenu dans la scène 1 de l'acte I de la tragédie

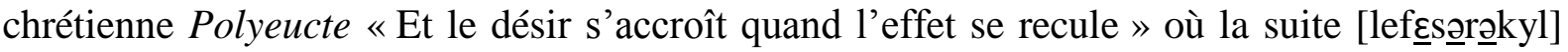
peut être comprise «les fesses reculent». Sur le même modèle, on entend parfois la formule

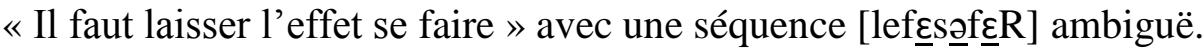

Une des sources importantes est le domaine de la communication, en particulier la publicité. Les slogans doivent retenir l'attention, se graver dans la mémoire, séduire, convaincre... Parmi les procédés mis en œuvre et bien étudiés par B.-N. Grunig (1990), les jeux de mots sont en bonne place. Ils ont pour intérêt de procurer du plaisir et de bien disposer les récepteurs ciblés à l'égard de l'auteur du message et de ce qu'il cherche à promouvoir. L'aspect colludique, qui mêle inextricablement le jeu et la connivence, est un facteur important de la réussite du message.

Cet aspect joue aussi à plein dans certains types de discours comme les discours politiques, mais à la différence du cas précédent où les néologismes ludiques sont au service de l'éloge, ceux qui apparaissent dans le discours politique servent plutôt à déconsidérer des adversaires ou des idées que l'on combat. Les hommes politiques mettent ainsi les rieurs de leur côté et un nouveau jeu de mots, bon ou mauvais, vaut souvent autant qu'une argumentation, même s'il ne prouve rien : il a le mérite de frapper les esprits et de bien disposer les citoyens à l'égard de celui qui le produit ou du moins de disposer de manière négative envers celui qui en fait les frais.

Les conversations de la vie courante peuvent également secréter des néologismes ludiques en fonction des capacités linguistiques et culturelles des interlocuteurs, dans un certain nombre d'échanges de la vie quotidienne. Ils relèvent de la fonction phatique de Jakobson, en tissant des liens de complicité du fait qu'ils se fondent sur le partage d'un bagage lexico-culturel commun.

\subsection{Les fonctions imputables dans le Faisceau Causal ${ }^{30}$ se mêlent ou se surajoutent}

Il est difficile de ne pas parler par anticipation des fonctions attribuées ou attribuables à ces néologismes ludiques quand on parle des situations d'énonciation, mais il n'y a pas de

\footnotetext{
${ }^{30}$ Le Faisceau Causal* $\left(\mathrm{FC}^{*}\right)$ est ce qu'un auditeur / lecteur attribue comme motivations à un énoncé qu'il interprète et répond aux questions qu'il se pose de savoir pourquoi le locuteur prend la parole, dit ce qu'il dit, et avec les mots qu'il emploie, et pas d'autres. La distinction et l'inévitable écart entre le Faisceau Causal (FC, constitué de pressions $\mathrm{P}$ ) à l'origine effective de l'énoncé d'un locuteur (dont il ne peut jamais avoir une conscience complète et juste, d'où la fuite du sens à gauche) et les hypothèses construites par les récepteurs interprétants à propos des causes de cet énoncé par le locuteur (le FC*, constitué de pressions $\mathrm{P}^{* *}$ ) constituent une des sources de la fuite du sens à droite. L'interprétant est en effet soumis dans son travail d'interprétation à des pressions $\mathrm{P}^{*}$ qui influent sur sa construction du sens. Pour plus de précisions à ce sujet, voir Grunig 1985.
} 
relations bi-univoques entre ces deux ensembles et il est aussi difficile de les traiter simultanément.

\subsubsection{Accroche}

La fonction d'accroche semble quasiment toujours présente: un néologisme est souvent fait pour se faire remarquer, a fortiori un néologisme ludique. Comme aucune charge sémantique n'est disponible, emmagasinée dans la mémoire à la suite de rencontres antérieures, le récepteur-interprétant doit nécessairement se livrer à un calcul du sens pour un signifiant qu'il découvre. Dans le cas d'un néologisme sémantique ou d'emploi, c'est l'inadéquation de ce qu'il a en tête qui le conduit à construire une interprétation qui convienne en se fondant sur le contexte et sur l'analyse morphologique. C'est particulièrement vrai dans le cas des remotivations ou des doubles lectures conventionnelle et compositionnelle. Ce travail attire l'attention sur le mot ou l'expression néologiques et, par extension, sur le message qui l'inclut.

\subsubsection{Connivence}

La connivence avec l'interlocuteur ou les interlocuteurs est aussi quasiment toujours présente sauf dans les cas où le néologisme ludique est une provocation, voire une agression envers l'interlocuteur. Les néologismes ludiques se fondent en effet sur un savoir partagé, avec le plaisir de la conscience de l'appartenance à un même groupe, ainsi que sur la reconnaissance que l'on a envers son interlocuteur de se faire une bonne image de soi-même, pour avoir surmonté victorieusement l'épreuve de compréhension d'un mot inconnu et d'identification de sa fonction ludique. L'inextricable mélange du jeu et de la connivence a conduit Camille Vorger (2011) dans un article et dans sa thèse sur le slam à créer le concept et le mot colludique (v. 1.1.3.). Ce qui est vrai du slammeur et de ses auditeurs l'est aussi dans d'autres types d'échanges, chaque fois qu'un certain type de lien est institué entre locuteurs et interlocuteurs et fondé sur le comique de mots. Mais le jeu peut également être plus complexe quand la connivence avec les membres d'un groupe se fait au détriment d'autres personnes non membres du groupe et exclus de la connivence. On l'a vu aussi (en note 6) pour les blagues où un des récepteurs ne comprend pas.

\subsubsection{Arme de combat}

Dans des situations d'affrontement, le recours aux néologismes ludiques met les rieurs de son côté. C'est vrai, comme on l'a vu, du discours politique quand on veut discréditer quelqu'un (ou quelque chose) en cherchant à l'isoler face au groupe des interlocuteurs s'associant au locuteur (cf. supra capitaine de pédalo, serial menteur, etc.). Dans un autre domaine, celui de la médecine et de la santé, un urologue nomme chauffe-prostate le traitement par la chaleur (qu'il juge par là même inefficace) de l'adénome de la prostate.

C'est encore dans un autre domaine, celui de la langue, que des néologismes servent d'armes à certains locuteurs, pas nécessairement puristes d'ailleurs, qui se gaussent des formulations néologiques prétentieuses d'autrui en faisant une surenchère qui dévalorise ipso facto les procédés mis en œuvre et leurs résultats. Philippe Meyer dans ses chroniques procède parfois ainsi. Outre fruitdemerologue et institut de légumologie appliquée etc., déjà cités, il se moque des auteurs d'un carton d'invitation à un «buffet déjeunatoire » en créant « déjeuner buffétatif » et « réception digestatoire »...

Les néologismes beautisme, votage ont été fabriqués par dérision par les adversaires de Ségolène Royal pour se gausser de son bravitude (prononcé au pied de la Muraille de 
Chine) et du coup mettre en doute sa capacité à gouverner faute de maitriser la langue française. Mais les fautes de français de son rival pendant cette campagne électorale de 2007 n'ont pas trouvé de censeurs aussi rigoureux.

\subsubsection{Argument de vente}

C'est comme argument de vente qu'apparaissent la plupart des néologismes ludiques des slogans publicitaires : il faut bien disposer l'acheteur potentiel et faire en sorte qu'il mémorise le produit (matériel ou non) afin de le faire choisir de préférence à d'autres concurrents. Parfois ce sont les noms de marque ou de produit qui sont eux-mêmes néologiques, mais peu sont des jeux de mots (V. néanmoins le slogan Javel dire à tout le monde et le nom Tout en camion (paronyme de Tout Ankh Amon) d'une entreprise de carrosserie spécialisée dans les poids lourds, ou encore le nom du produit destiné à nettoyer les lunettes pour bien voir, $V u$, avec le slogan $V u$, j'avais pas $v u^{31}$ ).

\subsubsection{Provocation}

La provocation peut aussi se manifester par des jeux lexicaux. Ainsi un des personnages des Voyageurs de l'impériale d'Aragon déclare son mépris pour ce qui est généralement valorisé, la lignée dont on est issu, surtout quand on est aristocrate, comme c'est le cas, en disant à son interlocuteur «quant aux ancêtres, permettez que je m'en torboyaute avec quelque solennité » s'en prend à la fois à la tradition qui fait vénérer ses pères, mères et leurs ascendants, à son interlocuteur, et à ses aïeux, avec ce mot-valise combinant le verbe familier (et vieilli maintenant) se boyauter « rire » et l'expression, familière aussi, se tordre de rire. La mot-valisation mettant en jeu des lexies familières et la rupture de ton entre ce vocabulaire familier et la forme soignée, voire soutenue, de l'énoncé créent un effet humoristique, comme le font souvent les décalages stylistiques.

\subsection{La durée de vie des néologismes ludiques}

Liée aux conditions d'énonciation, la durée de vie des néologismes ludiques est variable. Cela va de l'hapax conversationnel, ponctuel et rarement réutilisable, sauf comme citation (incontinente pour une voiture « qui a des fuites d'huile »; voir aussi Fradin 2000), à l'introduction dans la langue courante et la perte de la néologicité. Ce dernier cas est rare ${ }^{32}$ à la différence du premier qui est le plus fréquent, avec néanmoins les citations qui peuvent être faites de l'occurrence ${ }^{33}$ originelle. Mais on a tendance à oublier des situations intermédiaires, pourtant bien attestées.

Il y a d'abord le cas des récepteurs multiples d'une source unique : les néologismes littéraires (ludiques ou non, de Rabelais, Laforgue, Aragon...) sont produits une fois par l'auteur mais ce sont des milliers, voire des millions de lecteurs qui les découvrent et pour qui ce sont toujours des néologismes, et cela à travers le temps. Les néologismes ludiques de Rabelais - comme ceux de la scène où Frère Jean des Entommeures combat l'armée de

\footnotetext{
${ }^{31}$ Avec un jeu sur la conjugaison : maintenant que j'ai $\mathrm{Vu}$ (où ai est le présent du verbe avoir "posséder», mais il pourrait être l'auxiliaire du passé composé de voir), je vois. Et le slogan se terminait par une adresse, familière, aux spectateurs-auditeurs : Vu ? au sens de « compris ».

${ }^{32}$ Citons néanmoins le cas de la remotivation par les prisonniers français, ignorants que c'était la véritable étymologie, de vasistas. Aux questions des soldats allemands qui posaient la question Was ist das, ils s'amusaient à répondre : petite fenêtre.

${ }^{33}$ Les données amassées dans la base Neologia confirment le fréquent statut d'hapax de nombre des néologismes ludiques, qui ne circulent guère, pendant une période, que comme citations.
} 
Picrochole qui s'en prend aux vignes de son monastère - le sont toujours pour les lecteurs du $21^{\mathrm{e}}$ siècle, cinq siècles après leur création :

« es autres délochait les spondyles du col, es autres démoulait les reins, avalait le nez $[\ldots]$ décroulait les omoplates, sphacelait les grèves, dégondait les ischies, débezillait les faucilles » Gargantua ch. $\mathrm{XXVII}^{34}$ ) .

S'ils restent des hapax du point de vue de la production, ils n'ont pas le même statut que des hapax conversationnels qui peuvent disparaître à tout jamais si personne ne les consigne.

Proches de ces néologismes littéraires pour ce qui est de la diffusion, il y a les créations des humoristes : s'ils en sont les créateurs et (quasiment) les seuls utilisateurs, ils les profèrent chaque fois qu'ils font leur sketch en public, c'est-à-dire des centaines de fois, devant des publics différents, sans compter que ces sketchs peuvent être diffusés et rediffusés à la radio, à la télévision ou être disponibles sur des supports audio-visuels.

C'est donc une palette de situations diversifiées qui se manifestent pour la durée des néologismes ludiques : une occurrence unique, une occurrence reprise comme citation, une occurrence avec des milliers d'actes de réception et d'interprétation étalés dans le temps et dans l'espace, des occurrences multiples par répétition par leur auteur, une diffusion éventuelle dans le corps social pour une minorité d'entre eux, du moins pendant une certaine période (comme c'est le cas pour des slogans publicitaires ou des créations d'hommes politiques : le capitaine de pédalo est repris à l'envi par les journalistes et les opposants au Président de la République).

\section{Conclusion}

Si tous les jeux de mots ne sont pas néologiques et tous les néologismes pas des jeux de mots, l'intersection entre la néologie et les jeux de mots est un domaine assez vaste qui se présente sous des aspects divers. Cette contribution avait pour objectif modeste de présenter plusieurs de ces aspects. L'auteur espérait au début de sa recherche dans le domaine associer des points de vue divers pour monter des convergences entre l'identification des matrices lexicogéniques, les types de situations énonciatives où ils apparaissent, les fonctions que leurs créateurs entendent leur faire tenir, du moins dans l'interprétation que s'en font les récepteurs qui ne manquent pas de s'interroger à ce sujet, et enfin la durée de vie de ces néologismes ludiques. La complexité des faits l'a conduit à abandonner cet objectif, sans doute trop ambitieux, mais des recherches ultérieures devraient permettre néanmoins d'opérer des regroupements ou de dégager des tendances. Autant dire que beaucoup reste à faire dans le domaine. Néanmoins l'examen sans a priori de données recensées dans la base Neologia et d'autres plus anciennes montre que certaines matrices sont plus utilisées que d'autres dans la création de néologismes ludiques : la part de la morphologie extragrammaticale est importante par exemple. Par ailleurs cet aspect ludique est mis à profit dans la communication pour des fonctions diverses dont certaines sont bien identifiables.

\section{Bibliographie}

Benveniste Émile, [1966] 1974, «Formes nouvelles de la composition nominale », Problèmes de linguistique générale II, NRF-Gallimard, p. 163-176.

\footnotetext{
${ }^{34}$ Le manuel «Lagarde et Michard » donne comme explications en notes : « démettait les vertèbres », « disloquait les reins », « défonçait le nez », «meurtrissait les jambes », « déboîtait les hanches » et « rompait les bras ». Le manuel Magnard XVIe-XVIIe siècles de Chrisian Biet et alii donne également une traduction de ces néologismes.
} 
Biet Christian, Brighelli Jean-Paul et Rispail Jean-Luc, 1981, XVIe - XVIIe siècles (Collection Textes et contextes), Magnard.

Corbin Danielle, 1987, Morphologie dérivationnelle et structuration du lexique, 2 vol., Tübingen, Max Niemeyer Verlag.

Cusin-Berche Fabienne, 2003, « Des mots qui bougent : le lexique en mouvement », Les mots et leurs contextes, Presses Sorbonne Nouvelle.

Fradin Bernard, 2000, «Combining forms, blends and related phenomena », In Doleschal, U., Thornton, A. M. (eds). Extragrammatical and Marginal Morphology. München: Lincom Europa, p. 11-59.

Fradin Bernard, 2003, Nouvelles approches en morphologie, PUF.

Fradin Bernard, Montermini Fabio et Plénat Marc, 2009, «Morphologie grammaticale et extragrammaticale » in Fradin et al. éds., Aperçus de morphologie du français, Saint Denis, Presses Universitaires de Vincennes, p. 21-45.

Freud Sigmund, Le mot d'esprit et ses rapports avec l'inconscient, Paris, Gallimard 1969 [1905]

Grésillon Almuth, 1985, «Le mot-valise, un monstre de langue?» La linguistique fantastique, Auroux-Chevalier éds., p. 245-259.

Gross Gaston, 2012, Manuel d'analyse linguistique, Presses du septentrion.

Grunig Blanche-Noëlle, 1990, Les mots de la publicité, Paris Presses du CNRS.

Grunig Blanche-Noëlle et Grunig Roland, 1985, La fuite du sens, Hatier-CREDIF.

Guiraud Pierre, 1976, Les jeux de mots, P.U.F., Que sais je ?, $\mathrm{n}^{\circ} 1656$.

Hesbois Laure, [1986] 1988, Les jeux de langage, Les Presses de l'Université d'Ottawa.

Holmes Anne, 1993, Jules Laforgue and poetic innovation, Oxford Univerty Press, p. 34-35.

Lagarde et Michard, XVIe siècle, Les grands auteurs du programme. Anthologie et Histoire littéraire, Bordas, $1^{\text {ère }}$ éd. 1948, nombreuses rééditions.

Le Pesant Denis et Mathieu Colas Michel, 1994, «Introduction aux classes d'objets », Langages $\mathrm{n}^{\circ} 115$, p. 6-33.

Léturgie Arnaud, 2011, «Un cas d'extragrammaticalité particulier : les amalgames lexicaux » Linguistica, 51, p. 87-104.

Léturgie Arnaud, 2012, L'amalgamation lexicale en français : approches lexicologique et morphologique 'Vers une grammaire de l'amalgamation lexicale en français' (thèse soutenue à Cergy le 23 décembre 2012.

Ludi Georges, 1983, "Aspects énonciatifs et fonctionnels de la néologie lexicale », in TRANEL $\mathrm{n}^{\circ}$ 5, juin 1983, p. 105-130.

Matoré Georges, 1952, «Le néologisme : naissance et diffusion », Le français moderne $20^{\mathrm{e}}$ année $\mathrm{n}^{\circ} 2,1952$, p. 87-92.

Mounin Georges, 1990, «Quelques observations sur le lexique français d'aujourd'hui », Europe $\mathrm{n}^{\circ} 738$, octobre 1990, pp. 10sq

Reboul Sandrine, 1996, «Les mots-valises : de la création ludique à la dénomination », Akten des Kolloquiuns im Radmen des Erasmus-Netzes der Univ. Paris X-Nanterre, Duisburg und Trier 12-13 Mai 1995, in Herrmann Michael (ed.) ; Holz Karl (ed.). Sprachspiele und Sprachkomik/Jeux de mots et comique verbal. Frankfurt : Peter Lang, p. 197-219.

Sablayrolles Jean-François, 1993, «Fonctions des néologismes », in Cahiers du C.I.E.L (Centre Interlangue d'Études en Lexicologie, UFR EILA, Université Paris 7), «Lexique et construction du discours », p. 53-94.

Sablayrolles Jean-François, 2000, La néologie en français contemporain, Examen du concept et analyse de productions néologiques récentes, coll. Lexica mots et dictionnaires, Paris, Champion. 
Sablayrolles Jean-François, 2003, « Nouveauté, reprise et détournement dans les mots et slogans publicitaires », in L'invention verbale en français contemporain, Les cahiers $d u$ CIEP, éd. Didier, p. 36-45.

Sablayrolles Jean-François, à paraître, « Néologie et analogie », Actes du colloque Langage et analogie. Figement et argumentation, Tozeur 3-5 octobre 2012.

Vorger Camille, 2011a, «Le slam est-il néologène ? », Neologica 5, Classiques Garnier, p. 77-95.

Vorger Camille, 2011b, Poétique du slam: de la scène à l'école (Néologie, néostyles et créativité lexicale), thèse dirigée par Francis Grossmann et Dominique Abry et soutenue à Grenoble le 23 novembre 2011.

Sources primaires (auteurs à l'exclusion des publicitaires et hommes politiques)

Aragon Louis, 1948, Les Voyageurs de l'impériale, Gallimard.

Chiflet Jean-Loup, 1999, Le cafard laqué. Les mots-portemanteaux, Paris, Mots et Cie.

Chiflet Jean-Loup, 2002, Mais que fait l'Académie? Le dictionnaire des mots qui devraient exister, Paris, Mots et Cie.

Créhange Alain, 2004, Le pornythorinque est un salopare, éditions Mille et une nuits.

Devos Raymond, Sketchs.

Finkielkraut Alain, 1979, Ralentir mots-valises, Seuil.

Laforgue, Les complaintes, Poésie-Gallimard édition de 1979, et correspondance (Lettres à sa sœur).

Meyer Philippe, Chroniques parues dans collection Point actuel au Seuil : 1990, Heureux habitants de l'Aveyron et des autres départements français ; 1990, Ça n'est pas pour me vanter; 1991, Nous vivons une époque moderne; 1993, Dans le huis clos des salles de bain ; 1993, Chroniques matutinales.

Rabelais, Gargantua.

Renaud, « dès que le vent soufflera », album Morgane de toi, 1983.

Simon Claude, 1960, La Route des Flandres, Les Éditions de minuit.

Sol (Marc Favreau dit), 1982. Je m'égalomane à moi-même, Stanké.

Vian Boris, chanson : « la complainte des arts ménagers ».

Vian Boris, 1947. L'Automne à Pékin, éditions du Scorpion ( $1^{\text {ère }}$ éd., en Livre de poche depuis).

Sites

http://www.cledut.net/xylo.htm (défense et illustration de la xyloglosse)

Appendice : liste des néologismes ludiques cités, par ordre alphabétique

altesse de l'air 1.1.2. sketch de Sol (Marc Favreau) «L'hôtesse de l'air', Je mégalomane à moi-même, Stanké, 1982

avaler (le nez) 2.3. Rabelais, Gargantua, XXVII, 1534

biberonite 1.2.1.3., Aristophane, Les guêpes, trad. de Debidour (reprise en Folio)

brèves de pouvoir 1.1.3. Télérama, 30.10.2010 (rediffusion d'un documentaire de 2008)

brèves de wagon 1.1.3. Télérama 2004 probablement

buffétatif 2.2.3. Philippe Meyer, Nous vivons une époque moderne, Points actuel, Seuil, 1991

bulance (la) 1.1.2., Hara-Kiri, $\mathrm{n}^{\circ}$ 10, 10 mars 1993 (dans une bande dessinée)

cactus dans le myocarde, 1.1.2. Hara-Kiri, $\mathrm{n}^{\circ}$ 10, 10 mars 1993 (dans une bande dessinée) 
canon à patates 1.2.1.2. Boris Vian, Chanson La complainte des arts ménagers capitaine de pédalo 1.2.1.2. ; 2.2.3. et 2.3. Jean-Luc Mélenchon, 13 novembre 2011

castoche 1.1.1. publicité pour Castorama, 2009

Chaban d'Estaing 1.1.1. dit par un homme politique de gauche en 1974

chauffe-prostate 2.2.3. Le Monde 19.4.1991 et 31.3.1993 (A Le Duc urologue puis J.-Y. Nau, journaliste)

dans ma Ford intérieur 1.1.2., Frédéric Dard (San Antonio), récurrent, cité par Delphine Allerat, dans son mémoire de maîtrise, Étude du lexique dans l'œuvre de Frédéric Dard, Université de Limoges, 1999

débeziller 2.3. Rabelais, Gargantua, XXVII, 1534

décrouler 2.3. Rabelais, Gargantua, XXVII, 1534

degonder 2.3. Rabelais, Gargantua, XXVII, 1534

délocher 2.3. Rabelais, Gargantua, XXVII, 1534

démouler (les reins) 2.3. Rabelais, Gargantua, XXVII, 1534

dépasser les bornes 1.1.3. publicité pour le Peugeot Partner diesel, septembre 2001

digestatoire 2.2.3. Philippe Meyer, Nous vivons une époque moderne, Points actuel, Seuil, 1991

égalomane à moi-même (je m') 1.1.2., titre d'un recueil de sketches de Sol, (Marc Favreau), Stanké, 1982

élevache 1.1.1. exemple donné par Bernard Fradin 2000

emmaillobêté 1.1.1. Aristophane, Les nuées, traduction proposée par Jean Taillardat en cours (1974)

émolument (mon) 1.1.4. Alphonse Allais, anecdote souvent rapportée, mais sans date ni œuvre

être les dindons de la crise 1.1.3. tract étudiant à Paris 13 en 2010 ou 2011

fracture du myocarde 1.1.2., Titre du film Jacques Fansten, 1990

fruitdemerologue 1.2.2.2. et 2.2.3. Philippe Meyer, Nous vivons une époque moderne, Points actuel, Seuil, 1991

Giscard Delmas 1.1.1. dit par un homme politique de gauche en 1974

Gyptiens (les) 1.1.2. Sol, Marc Favreau, cité par Laure Hesbois ([1986] 1988)

incontinente (voiture) 2.3. conversation, au milieu des années 1980

institut de légumologie appliquée 1.2.2.2. et 2.2.3. Philippe Meyer, Nous vivons une époque moderne, Points actuel, Seuil, 1991

javel dire à tout le monde 1.1.2., spot publicitaire télévisé pour l'eau de Javel, 10.07.1996

je repartira 1.1.4., " dès que le vent soufflera », album Morgane de toi, 1983

je travaille, tu travailles, il travaille, nous travaillons, vous travaillez, ils profitent 1.1.4. affiche politique (PCF ou CGT ?) dans les années 1970 ?

kératocéphale 1.2.2.2. site xyloglosse : http://www.cledut.net/xylo.htm

lance-hostie 1.2.1.1. Boris Vian, L'automne à Pékin, 1947

maasricheur 1.1.1. Jean-Pierre Chevènement, Le Monde, 19 mai 1992

mec plus ultra 1.1.2. conversation, milieu des années 1980

mensongite 1.2.1.3., Marianne, 27 juillet 2013

ne pas avoir la lumière à tous les étages 1.1.3. conversation, début des années 2010

ne pas être branché haut débit 1.1.3. Télérama, 22 décembre 2004

ne pas faire du huit megabits 1.1.3. Télérama, 22 décembre 2004

nesthésie 1.1.2., Hara-Kiri, $\mathrm{n}^{\circ}$ 10, 10 mars 1993 (dans une bande dessinée)

nounoursothérapie 1.2.2.1., Valeurs actuelles, revue de la MGEN

nous nous en allerons 1.1.4. « dès que le vent soufflera », album Morgane de toi, 1983

pastille Lajoignie 1.1.2., conversation surprise, milieu des années 1990

pommade «jus de pomme » 1.2.1.4., exemple emprunté à Danielle Corbin 1987 
pyrowoman 1.1.1. conversation, début des années 1990

ratatine-ordure 1.2.1.1. Boris Vian, Chanson La complainte des arts ménagers

remonter (la mer démontée) 1.2.1.4. Sketch de Raymond Devos « La mer », 1956

ripoublique 1.1.1., Jean-Marie Le Pen, 15.04.1988

ronronthérapie 1.2.2.1. Marianne, 17 août 2013

sangsuel 1.1.1., Laforgue, Complaintes,

sardin (un) 1.1.4. conversation surprise dans le métro, début des années 1990

sarkhollandisation 1.1.1. Bayrou, 17.02.2012

self made mélomane 1.1.1. Daniel Schick, France Musique, 13 mars 1993

serial menteur 1.1.1. et 2.2.3. Jean-Marie Le Pen, 18.02.2002

serial papa 1.1.3. Direct marin, 1.12.2011

sortir son couteau pour un oui pour un non 1.1.3., page publicitaire pour les scouts, Télérama, été 2001

sphaceler 2.3. Rabelais, Gargantua, XXVII, 1534

sur ces entrefesses 1.1.2. Frédéric Dard (San Antonio), cité par Delphine Allerat, dans son mémoire de maîtrise, Étude du lexique dans l'œuvre de Frédéric Dard, Université de Limoges, 1999

sur ces entremiches 1.1.2. Frédéric Dard (San Antonio), cité par Delphine Allerat, dans son mémoire de maîtrise, Étude du lexique dans l'œuvre de Frédéric Dard, Université de Limoges, 1999

torboyaute (je m'en) 2.2.5. Aragon, Les voyageurs de l'impériale

Tout en camion 2.2.4., Nom d'une entreprise de carrosserie du Loiret, spécialisée dasn les camions

vectorologue de l'écriture 1.2.2.2. Philippe Meyer, Nous vivons une époque moderne, Points actuel, Seuil, 1991

vélocipédophile 1.2.2.2. Philippe Meyer, Ça n'est pas pour me vanter, Points actuel, Seuil, 1991

Vu, j'avais pas vu 2.2.4. Slogan publicitaire télévisé pour produit de nettoyage de lunettes $\mathrm{Vu}, 30.07 .2001$

xyloglosse 1.2.2.2. site http://www.cledut.net/xylo.htm

vasistas / Was ist das ? 2.3. anecdote racontée par un ancien prisonnier de guerre (1939-1944) en Allemagne

waterbraguette 1.1.1. Libération, 23.01.1998 